\title{
DIVERSIDAD MORFOLÓGICA Y CONCENTRACIÓN DE PROTEÍNA DE LUPINUS SPP. EN LA REGIÓN CENTRO-ORIENTAL DEL ESTADO DE PUEBLA, MÉXICO
}

\author{
LuZ del Carmen Lagunes-Espinoza ${ }^{1,4}$, Javier López-Upton ${ }^{2}$, \\ Eustolia García-LóPez ${ }^{1}$, Jesús Jasso-Mata ${ }^{2}$, \\ Adriana Delgado-Alvarado ${ }^{3}$ y Gabino García de los Santos ${ }^{2}$
}

${ }^{1}$ Colegio de Postgraduados, Campus Tabasco, Periférico Carlos A.

Molina s/n, 86500 H. Cárdenas, Tabasco, México.

${ }^{2}$ Colegio de Postgraduados, Campus Montecillo, km. 36.5 carretera

México-Texcoco, 56230 Montecillo, Edo. de México, México.

${ }^{3}$ Colegio de Postgraduados, Campus Puebla, km. 125.5 carretera federal MéxicoPuebla, 72760 Santiago Momoxpan, San Pedro Cholula, Puebla, México.

${ }^{4}$ Autor para la correspondencia: lagunesc@colpos.mx

\section{RESUMEN}

El objetivo del presente estudio fue caracterizar la diversidad morfológica y determinar la concentración de proteína en semillas del género Lupinus (Leguminosae) en los ecosistemas agrícola y forestal de la región centro oriental del estado de Puebla. Las poblaciones de Lupinus se localizaron entre 2600 y 4000 m s.n.m., en suelos con pH que varía de ácido (4.7) a neutro (7.1), con elevados contenidos de materia orgánica (> 7.8\%) y bajos niveles de $\mathrm{K}^{+}$intercambiable $\left(<6 \mathrm{cmol}^{+} \mathrm{g}^{-1}\right)$. Dichas poblaciones mostraron una gran variabilidad morfológica, resultado de la diversidad de los representantes de este género en el área de estudio. Cuatro especies fueron identificadas con base en sus características morfológicas e intervalo altitudinal: L. campestris, localizada entre 2600 y $3052 \mathrm{~m}$ sobre el nivel del mar, en bordes de cultivos de maíz y haba o áreas con bosque de Juniperus deppeana, Pinus spp. y Abies religiosa; L. exaltatus se encontró entre 2778 y $2865 \mathrm{~m}$ de altitud en bosques de $P$. montezumae; $L$. hintonii a $3080 \mathrm{~m}$ en bosques de $A$. religiosa; y $L$. montanus a elevaciones superiores a los $3000 \mathrm{~m}$ en bosques de $P$. hartwegii, $P$. montezumae, $P$. rudis y $A$. religiosa. Se registraron concentraciones altas de proteína de la semilla entre y en las especies; la primera en promedio varió de 328 a $387 \mathrm{~g} \mathrm{~kg}^{-1}$ de materia seca (MS) y la segunda de $317 \mathrm{~g} \mathrm{~kg}^{-1}$ (una población de L. exaltatus) a $481 \mathrm{~g} \mathrm{~kg}^{-1} \mathrm{MS}$ (una población de L. campestris). 
Palabras clave: concentración de proteína, Leguminosae, Lupinus, maleza, México, morfología, Puebla.

\begin{abstract}
The present study aimed to characterize the diversity of Lupinus genus (Leguminosae) present in agricultural and forest ecosystems of eastern central region of the Mexican state of Puebla, and to estimate seed protein concentration in species identified. Natural populations were located from 2600 to $4000 \mathrm{~m}$ above sea level in soils with neutral (7.1) to acid (4.7) $\mathrm{pH}$, with high content of organic matter $(>7.8 \%)$ and low levels of exchangeable $\mathrm{K}^{+}\left(<6 \mathrm{cmol}^{+} \mathrm{g}^{-1}\right)$. Natural populations of Lupinus showed a great morphological variability result of diversity of species in this genus present in the study area. Four species were identified based on their morphological characteristics and altitudinal range: L. campestris, located at altitudes of 2600 to $3052 \mathrm{~m}$, along edges of corn and faba bean crops or natural vegetation areas in the presence of Juniperus deppeana, various species of Pinus and Abies religiosa; L. exaltatus from 2778 to $2865 \mathrm{~m}$ in forests of P. montezumae; L. hintonii at $3080 \mathrm{~m}$ associated with Abies religiosa; and L. montanus, at altitudes above $3000 \mathrm{~m}$ associated with $P$. hartwegii, $P$. montezumae, P. rudis and Abies religiosa. High concentration of seed protein was observed within and between species. Among species, the average protein concentration ranged from 328 to $387 \mathrm{~g} \mathrm{~kg}^{-1}$ of dry matter (DM). Between the species, the concentration ranged from $317 \mathrm{~g} \mathrm{~kg}^{-1}$ (a population of L. exaltatus) to $481 \mathrm{~g} \mathrm{~kg}^{-1} \mathrm{DM}$ (a population of L. campestris).
\end{abstract}

Key words: legume, Lupinus, Mexico, morphology, protein source, Puebla, weed.

\title{
INTRODUCCIÓN
}

El género Lupinus es conocido por su elevado contenido de proteína en las semillas y su capacidad regeneradora de suelos; comprende entre 220 y 230 especies (Lewis et al., 2005). Su mayor diversidad se localiza en el Hemisferio Occidental y sólo se conocen 12 nativas de la región mediterránea y del norte de África (Planchuelo, 1994; Gladstones, 1998). Las zonas montañosas de América Central y principalmente de México son consideradas como comarcas de mayor concentración de representantes de Lupinus (Dunn, 1984), donde se ha observado una gran heterogeneidad por la plasticidad fenotípica que presenta, los hábitos de crecimiento anuales y perennes, la habilidad para adaptarse a ambientes diversos y el alto grado 
de cruzamiento, lo que ha dificultado la delimitación taxonómica de sus especies (Planchuelo, 1994). Para nuestro país, Sousa y Delgado (1998) reportan alrededor de 65 especies de este género; de las cuales $\pm 60 \%$ son consideradas endémicas.

En México, las poblaciones naturales de Lupinus crecen en caminos, laderas de cerros, en bosques degradados y en ecosistemas naturales a altitudes que van desde el nivel del mar hasta más de $4000 \mathrm{~m}$. Geográficamente se distribuyen desde Baja California hasta Tamaulipas y al sur hasta Chiapas. No obstante, la mayor concentración de especies se registra en la Sierra Madre Occidental y el Eje Neovolcánico Transversal (Ruiz-Moreno et al., 2000). Para la última se han reportado diferentes representantes de Lupinus que crecen de manera natural a partir de 1300 m s.n.m., entre ellas L. leptophyllus Schltdl. \& Cham., L. montanus Kunth, L. versicolor Steud., L. potosinus Rose y L. uncinatus Schltdl. (Alderete-Chávez et al., 2008). En el Parque Nacional Pico de Orizaba, L. montanus es una de las especies dominantes del estrato herbáceo junto con Agrostis tolucensis y Muhlenbergia sp. (Vargas, 1984). Villaseñor y Espinosa (1998) citan a L. leptophyllus ("garbancillo") y L. campestris como malezas que crecen en orillas de caminos y parcelas en descanso en el Distrito Federal, Estado de México, Hidalgo, Jalisco, Puebla, Tlaxcala y Veracruz.

A nivel mundial, actualmente se cultivan cuatro especies de Lupinus: $L$. albus L., L. angustifolius L., L. luteus L. y L. mutabilis Sweet. Las mismas han sido mejoradas para su uso en alimentación animal y humana (Clements et al., 2005) y el contenido de alcaloides se ha reducido, siendo actualmente inferior a $0.03 \%$ (Huyghe, 1997). A diferencia de los lupinos de Europa, norte de África y de América del Sur, donde su cultivo para uso alimenticio data desde hace más de 4000 años, en México no existen reportes del mismo o del uso del grano o del forraje como alimento, debido probablemente a la preferencia de otras fuentes de proteína y al elevado contenido de alcaloides que les confieren un sabor amargo y son tóxicos (Przybylak et al., 2005; Bermúdez-Torres et al., 2009). No obstante, el género ha sido objeto de estudios sistemáticos en nuestro país para conocer la calidad nutrimental y toxicológica de algunas especies. Investigaciones sobre la calidad nutritiva de la semilla de L. campestris (Cruz et al., 1999; Rodríguez-Ambriz et al., 2005; JiménezMartínez et al., 2009) y L. exaltatus (Ruiz-López et al., 2006) han demostrado su alto potencial como fuente de proteína para ser utilizados como complemento en la alimentación humana y animal, previa eliminación de alcaloides por medios físicos (Jiménez-Martínez et al., 2003).

Por otro lado, dada la alta capacidad de las especies de este género para fijar $\mathrm{N}_{2}$ atmosférico y solubilizar el $\mathrm{P}$, podrían ser también consideradas en la restauración ecológica de suelos degradados (Vance, 2001). 
En la porción del Eje Neovolcánico Transversal que atraviesa el estado de Puebla, los Lupinus forman parte del paisaje natural, y para promover su aprovechamiento sustentable se realizó un estudio exploratorio con el objetivo de caracterizar la diversidad de especies del género a nivel morfológico y de concentración de proteína en sus semillas en las zonas agrícola y forestal de los Valles de Libres y de Serdán del estado de Puebla, México.

\section{ÁREA DE ESTUDIO}

El área de estudio está situada en la parte centro-oriental del estado de Puebla en México, entre las coordenadas $18^{\circ} 52^{\prime} 23.0^{\prime \prime}$ y $19^{\circ} 54^{\prime} 43.9^{\prime \prime} \mathrm{LN}$ y $97^{\circ} 18^{\prime} 49.7^{\prime \prime}$ y $97^{\circ} 56^{\prime} 04.0^{\prime \prime} \mathrm{LW}$, a una altitud que varía de 1000 a más de $4600 \mathrm{~m}$ (Fig. 1). La temperatura promedio anual va de $22{ }^{\circ} \mathrm{C}$ en la partes bajas a $4{ }^{\circ} \mathrm{C}$ en las altas; la precipitación promedio anual acumulada va de 400 a $800 \mathrm{~mm}$ (Gutiérrez-Herrera et al., 2003). La zona está comprendida en la provincia del Eje Neovolcánico, como parte de las subprovincias de los lagos y volcanes del Anáhuac y Chiconguiaco. Las principales elevaciones son el Citlaltépetl (Pico de Orizaba) y el cerro La Negra con 5610 y 4621 m s.n.m., respectivamente.

El área destaca por su importancia agrícola y forestal en el estado de Puebla, actividades que han modificado el paisaje natural afectando probablemente la diversidad de la flora nativa. Zonas agrícolas, principalmente de maíz (Zea mays L.) y haba (Vicia faba L.), se observan hasta los 3200 m s.n.m., donde se utiliza la práctica de rotación de cultivos, que incrementa el contenido de materia orgánica en el suelo (Molumeli et al., 2008).

\section{MÉTODOS}

Se hicieron recorridos iniciales en los meses de octubre y noviembre de 2006 en el área de estudio, para identificar poblaciones de Lupinus, siguiendo un gradiente altitudinal a partir de 1800 a 4000 m (Fig. 1). Se consideró una población cuando los individuos del género Lupinus formaban grupos compactos pequeños o grandes con características morfológicas similares en el sitio. Durante estos viajes, en 2006, se colectaron ejemplares de Lupinus en floración y con presencia de vainas maduras de seis poblaciones en los municipios de Chalchicomula de Sesma y Tlachichuca. 
En los meses de octubre a diciembre del año 2007 se realizó una nueva colecta de ejemplares y semillas de Lupinus, identificándose 10 poblaciones en los municipios de Esperanza, Atzizintla, Chalchicomula de Sesma, Tlachichuca, Lafragua, Libres y Hueytamalco (Fig. 1). En este año, en cada población se registraron in situ, en

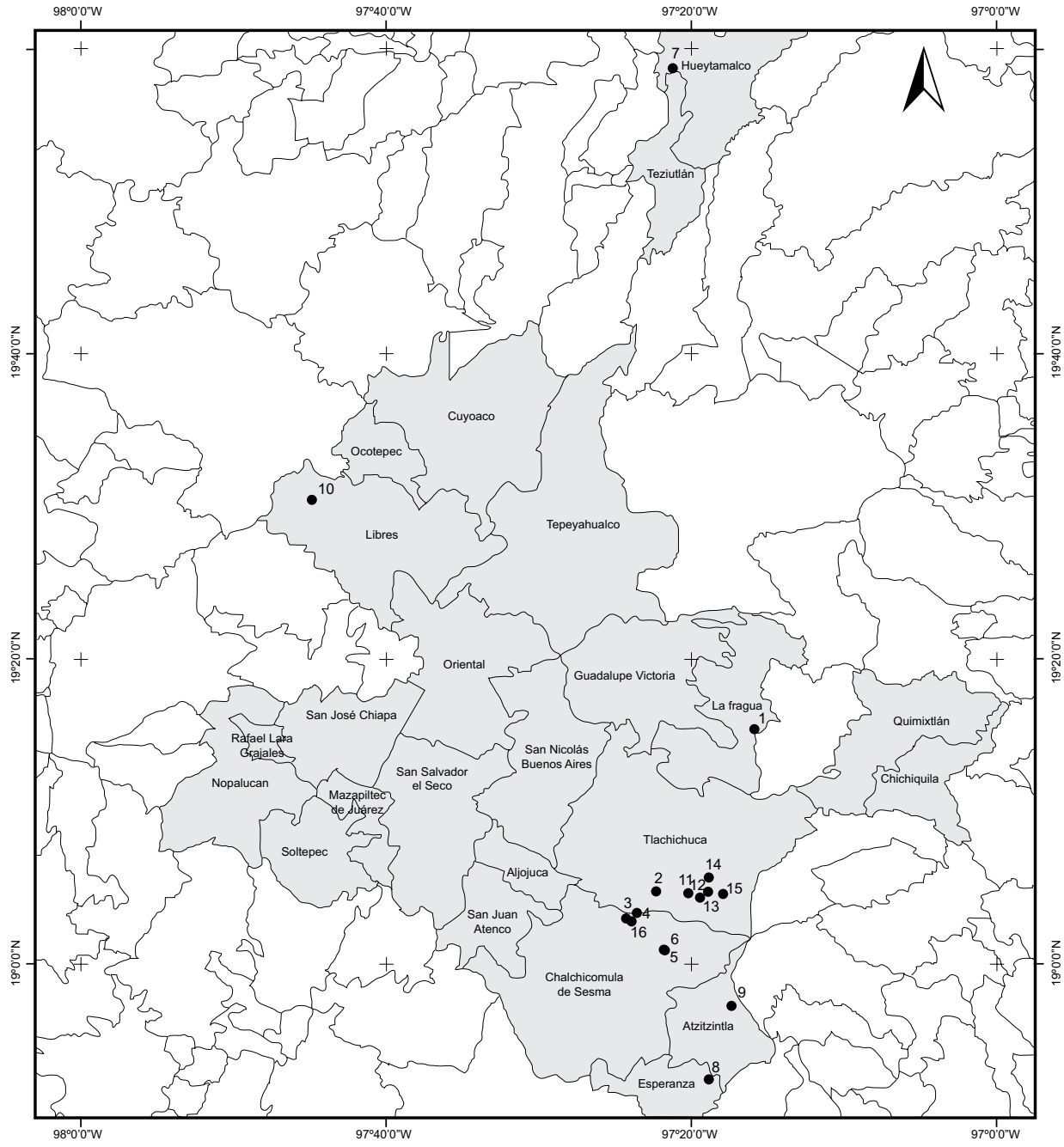

Fig. 1. Localización del área de estudio sombreada, indicando la ubicación de las poblaciones muestreadas del género Lupinus en la región de Valles de Serdán y Libres, Puebla. 1-10 (Colecta Octubre-Diciembre 2007); 11-16 (Colecta Noviembre 2006). L. montanus: 1, 6, 9 , 11, 12, 13, 14, 15; L. exaltatus: 2, 3, 4, 16; L. hintonii: 5: L. campestris: 7, 8, 10. 
5 a 20 plantas según el tamaño de la misma, las variables altura de planta, número de tallos por planta, diámetro del tallo principal, largo de la inflorescencia principal, número, largo y ancho de foliolos por hoja, número y tipo de tallos, presencia y tipo de estípulas, y largo y ancho de vaina. Además, se colectaron por población tres especímenes para su identificación taxonómica, la cual se realizó en primera instancia en el Herbario CSAT del Colegio de Postgraduados-Campus Tabasco, utilizando las claves taxonómicas y descripciones de Dunn (1979) y McVaugh (1987) para la Flora del Valle de México y Flora Novo-Galiciana, y la revisión del complejo de L. montanus de Dunn y Harmon (1977). Posteriormente, los ejemplares fueron enviados al herbario del Instituto de Biología de la Universidad Nacional Autónoma de México (MEXU) para la validación de su identidad taxonómica, en donde quedaron depositados.

Las coordenadas de los sitios de presencia y colecta de las poblaciones de lupino fueron registradas con un GPS (Garmin GPS 12 Map), se graficaron usando ArcView GIS 3.2 y se ubicaron sobre el mapa de la región de estudio.

La concentración de proteína cruda fue estimada a partir del contenido de nitrógeno total $(\% \mathrm{~N}$ x 6.25$)$ de las semillas obtenidas de diferentes plantas de cada localidad en 2007 (poblaciones 1 a 10), incluyendo, además, las de seis sitios muestreados en 2006 (poblaciones 11 a 16). Las semillas fueron previamente molidas y secadas a $60{ }^{\circ} \mathrm{C}$. Posteriormente $0.3 \mathrm{~g}$ de cada harina fueron pesados por triplicado y analizados por el método de semi-micro Kjeldahl digestión húmeda (Jones Jr. et al., 1991).

Muestras representativas de cada suelo donde se ubicaron las plantas de Lupinus se tomaron de $0-45 \mathrm{~cm}$ de profundidad, para determinar el $\mathrm{pH}$, el contenido de material orgánico $(\mathrm{MO}) \mathrm{y}$ el $\mathrm{K}^{+}$disponible.

El análisis estadístico de los datos consistió en el cálculo de medias y desviaciones estándar con la finalidad de conocer la variabilidad entre las poblaciones en cuanto a caracteres morfológicos y concentración de proteína. Para observar la variación espacial entre las poblaciones muestreadas en los sitios del año 2007 e identificar algunas variables que determinaran la separación de grupos, se realizó también un análisis de componentes principales, utilizando el paquete estadístico SAS versión 8.1 (Anónimo, 2000).

\section{RESULTADOS}

Distribución de poblaciones

Dentro de la flora nativa del área de estudio, las especies del género Lupinus forman parte de los ecosistemas agrícola y forestal y se les conoce con el nombre 
de "flor de San Juan" o "flor de San Pedro", y son utilizadas como ornamentales durante las fiestas religiosas y como forraje en seco para la alimentación de ovejas. Se localizan a partir de $2615 \mathrm{~m}$ s.n.m. en suelos con pH neutro a ligeramente ácido o ácido, con elevado contenido de materia orgánica y bajos niveles de $\mathrm{K}^{+}$intercambiable, este último disminuye al incrementarse la altitud (Cuadro 1). Estas condiciones de escasa fertilidad no impiden su crecimiento y desarrollo en el sotobosque o en claros de bosque. De acuerdo con Gladstones (1970), la mayoría de las especies del género crecen en suelos con $\mathrm{pH}$ inferior a 6.5 y exhiben un crecimiento reducido a valores de $\mathrm{pH}$ superiores a 6.5.

En el área de estudio el ecosistema forestal presenta efectos negativos del cambio de uso del suelo relacionados con la tala excesiva, la expansión de la frontera agrícola y la ganadería, lo que seguramente tiene un impacto en la biodiversidad de la región. Algunas especies arbóreas tienen importancia estratégica por su valor y abundancia en la zona, entre ellas Pinus montezumae y P. oaxacana. En este ambiente se observaron abundantes poblaciones de Lupinus en el sotobosque o en claros de bosque asociadas a diversas especies de Pinus, entre ellas P. oaxacana, P. patula, $P$. hartwegii, y a Pseudotsuga sp. y Abies religiosa a partir de los 2700 m s.n.m. en los municipios de Esperanza, Atzizintla, Chalchicomula de Sesma y Tlachichuca. En las zonas de transición entre bosques de pino, encino y Abies, aproximadamente a 2600 m s.n.m., Lupinus está asociado a Juniperus deppeana. A partir de los 3400 m s.n.m., éstas se localizan principalmente en bosques de Pinus rudis y P. montezumae, junto con varias especies de los géneros Abies, Senecio y Baccharis, estas dos últimas en el sotobosque, en el municipio de Tlachichuca. Asimismo, poblaciones abundantes fueron localizadas en bosques de $P$. montezumae asociadas con pastizales de Muhlenbergia sp. a altitudes que varían de 2778 a $2785 \mathrm{~m}$, en el mismo municipio de Tlachichuca. Los contenidos de MO en esta zona fluctúan de 7.8 a $10.2 \%$, el pH de 4.7 a 6.1 y los contenidos de $\mathrm{K}$ intercambiable son bajos.

En contraste con el ecosistema forestal, en el agrícola las poblaciones de $\mathrm{Lu}$ pinus fueron escasas, pero con un número elevado de individuos en cada sitio, y se localizaron principalmente en los márgenes de cultivos de maíz y/o de haba asociadas con Coreopsis sp., Galinsoga sp. y Lopezia sp. en suelos con pH ácido (5.7), ricos en MO (9\%).

Variación morfológica entre las poblaciones e identificación de especies

La amplia variación morfológica observada es el resultado de la diversidad de especies de este género en el área de estudio (Cuadro 2). Las plantas de las poblaciones 1, 6 y 9 presentaron también la mayor altura de todos los sitios colectados y la 5 mostró el más 
Cuadro 1. Altitud, características del suelo y especies vegetales asociadas a poblaciones del género Lupinus en la región de estudio durante 2006 y 2007.

\begin{tabular}{|c|c|c|c|c|c|c|}
\hline \multirow{2}{*}{$\begin{array}{l}\text { Altitud } \\
\text { m s.n.m. }\end{array}$} & \multirow[t]{2}{*}{ Población } & \multirow[t]{2}{*}{ Especie } & \multicolumn{3}{|c|}{ Características del suelo } & \multirow{2}{*}{ Principales especies asociadas } \\
\hline & & & $\mathrm{pH}$ & $\begin{array}{l}\mathrm{MO}^{*} \\
(\%)\end{array}$ & $\begin{array}{c}\mathrm{K}^{*} \\
\left(\mathrm{cmol}^{+} \mathrm{g}^{-1}\right)\end{array}$ & \\
\hline 2615 & 10 & L. campestris & 7.1 & 10 & 6 & Juniperus deppeana \\
\hline 2701 & 8 & L. campestris & 5.9 & 7.8 & 4 & $\begin{array}{l}\text { Árboles jóvenes de Pinus } \\
\text { patula, Pinus montezumae, } \\
\text { Pseudotsuga spp. }\end{array}$ \\
\hline 2778 & 16 & L. exaltatus & 5.7 & 9.2 & 3 & $\begin{array}{l}\text { Pinus montezumae, } \\
\text { Muhlenbergia sp. }\end{array}$ \\
\hline 2785 & 4 & $\begin{array}{l}\text { L. exaltatus } \\
\text { L. campestris }\end{array}$ & 5.7 & 9.2 & 3 & $\begin{array}{l}\text { Pinus montezumae, } \\
\text { Muhlenbergia sp. }\end{array}$ \\
\hline 2842 & 3 & $\begin{array}{l}\text { L. exaltatus } \\
\text { L. campestris }\end{array}$ & 5.8 & 10 & 3 & $\begin{array}{l}\text { Pinus montezumae, } \\
\text { Muhlenbergia sp. }\end{array}$ \\
\hline 2865 & 2 & L. exaltatus & 5.7 & 9 & 4 & $\begin{array}{l}\text { Cultivo de maíz y haba, } \\
\text { Coreopsis sp., Galinsoga } \\
\text { sp., Lopezia } \text { sp., Pinus } \\
\text { montezumae }\end{array}$ \\
\hline 2910 & 11 & L. montanus & 5.6 & 9.7 & 2 & Pinus montezumae \\
\hline 3052 & 7 & L. campestris & 5.5 & 8 & 2 & $\begin{array}{l}\text { Pinus montezumae y Abies } \\
\text { religiosa, Tagetes sp., } \\
\text { Eupatorium sp., Stevia sp. }\end{array}$ \\
\hline 3080 & 5 & L. hintonii & 5.6 & 8.4 & 3 & Abies religiosa \\
\hline 3080 & 6 & L. montanus & 5.6 & 8.4 & 3 & Abies religiosa \\
\hline 3115 & 1 & L. montanus & 4.7 & 7.8 & 2 & Límite con cultivo de maíz \\
\hline 3275 & 9 & L. montanus & 6.1 & 10.2 & 3 & Pinus hartwegii \\
\hline 3292 & 12 & L. montanus & 5.8 & 9.2 & 2 & $\begin{array}{l}\text { Pinus montezumae, } \\
\text { Muhlenbergia sp. }\end{array}$ \\
\hline 3423 & 13 & L. montanus & 4.9 & 9.2 & 2 & $\begin{array}{l}\text { Pinus montezumae, } \\
\text { Muhlenbergia } \mathrm{sp} .\end{array}$ \\
\hline 3471 & 14 & L. montanus & - & - & - & $\begin{array}{l}\text { Pinus rudis, Pinus } \\
\text { montezumae y Abies religiosa }\end{array}$ \\
\hline 3520 & 15 & L. montanus & - & - & - & $\begin{array}{l}\text { Pinus rudis, Pinus } \\
\text { montezumae y Abies religiosa }\end{array}$ \\
\hline
\end{tabular}

${ }^{*} \mathrm{MO}=$ Materia orgánica; $\mathrm{K}=$ Potasio 
Lagunes-Espinoza et al.: Diversidad morfológica y concentración de proteína de Lupinus, México

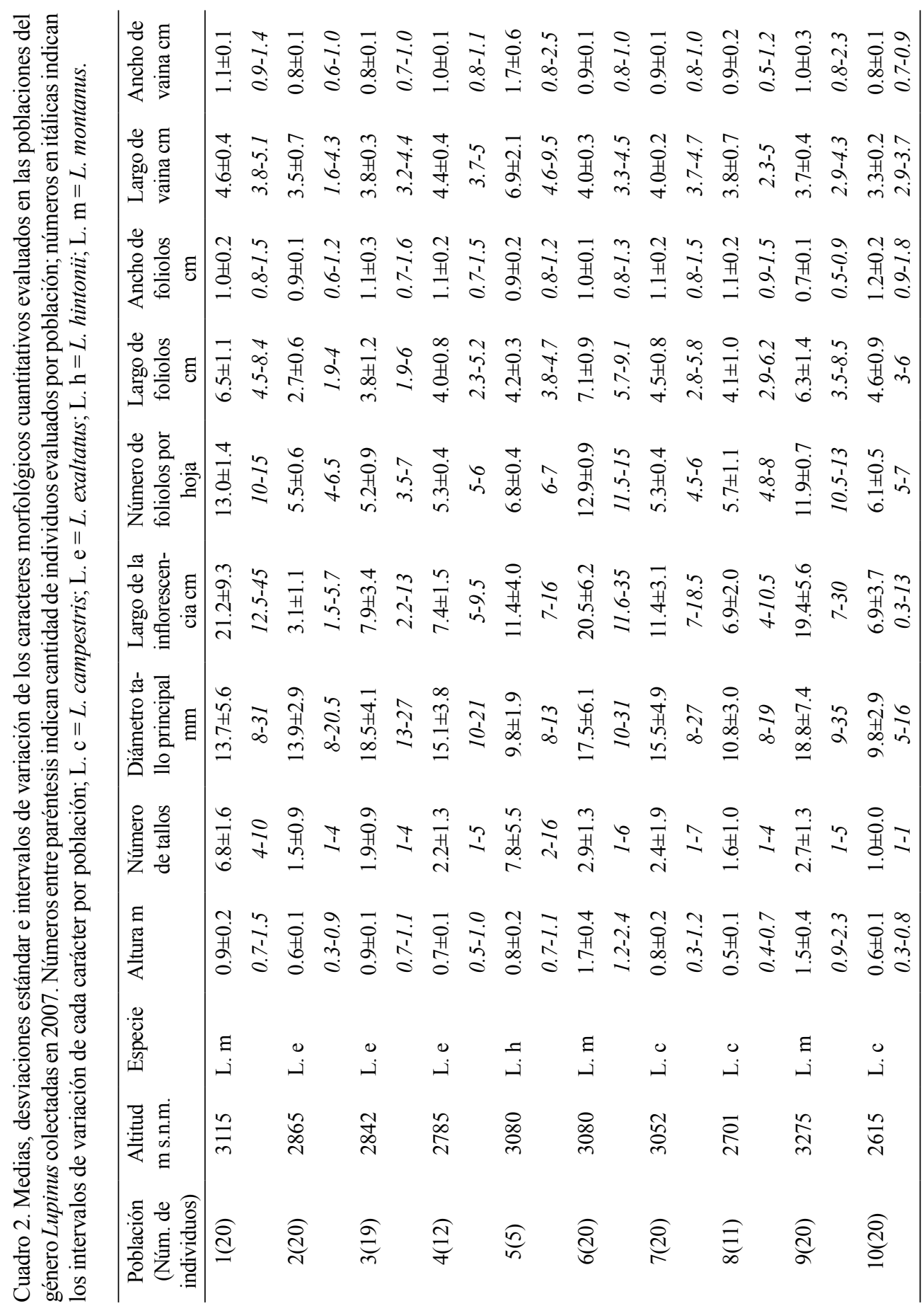


grande largo de vaina. Se registró también amplia diversidad morfológica entre los individuos al interior de las poblaciones. Los intervalos de variación más amplios fueron para el diámetro del tallo principal y el largo de la inflorescencia en las poblaciones 1, 6 y 9.

Para observar la variabilidad espacial de las poblaciones y su separación de acuerdo con los caracteres morfológicos cuantitativos y cualitativos (presencia y forma de estípulas, y tipo de tallo), se realizó el análisis de componentes principales (Fig. 2). Los tres primeros explicaron 69\% de la variación, correspondiendo 35\% al primero, $18.4 \%$ al segundo y $15.5 \%$ al tercero. Las variables que contribuyeron a la definición del componente principal uno (ACP1) fueron altura de planta, longitud de inflorescencia y número de foliolos por hoja; para el dos (ACP2) fueron largo y ancho de vaina y forma de estípulas, y para el componente tres (ACP3) largo de foliolos, forma de estípulas y tipo de tallo. En la figura 2 se observa que, con base en las características morfológicas evaluadas, las poblaciones 2, 3, 4 que corresponden a L. exaltatus se agrupan con la 7, 8 y 10 de L. campestris, y se separan de la 1,6 y 9 de L. montanus, por altura de planta, número de foliolos por hoja y forma de estípulas. La 5, de L. hintonii, diverge del resto al presentar diferencias marcadas en largo $\mathrm{y}$ ancho de vaina, tipo de tallos y forma de estípulas.

Estas características morfológicas, aunadas a las contenidas en las claves taxonómicas y descripciones consultadas (Dunn, 1979; McVaugh, 1987; Dunn y Harmon, 1977), permitieron identificar cuatro especies de Lupinus en los sitios muestreados en 2007. Todas las plantas recolectadas presentaron predominantemente flores de color morado-azuloso.

La población 5, que corresponde a la especie $L$. hintonii, presenta un intervalo de altura de planta que va de 70 a $110 \mathrm{~cm}$, diámetro del tallo principal de 8 a 13 $\mathrm{mm}$, con 6 a 7 foliolos por hoja, longitud y ancho de foliolos de 3.8 a $4.7 \mathrm{~cm}$ y 0.8 a $1.2 \mathrm{~cm}$, respectivamente, tallos delgados y sólidos, estípulas pequeñas, además de presentar el mayor tamaño de vaina $(4.6$ a $9.5 \mathrm{~cm})$. Sólo se estudiaron plantas de una localidad de esta especie, en la cual se encontró un bajo número de individuos, establecidos en suelos con $\mathrm{pH}$ de 5.6 en bosques de A. religiosa, a una altitud de 3080 $\mathrm{m}$ (Cuadro 1).

Las poblaciones 7,8 y 10 correspondieron a L. campestris. Se localizaron a partir de 2615 m s.n.m., asociadas a Juniperus deppeana, P. patula, Pseudotsuga spp., P. montezumae y A. religiosa, en bordes de terrenos cultivados con maíz hasta altitudes de $3052 \mathrm{~m}$. Éstas presentaron una altura promedio de planta de 0.8, $0.5 \mathrm{y}$ $0.6 \mathrm{~m}$, respectivamente, además de tallos sólidos, estípulas de $5 \mathrm{a} 12 \mathrm{~mm}$ de largo, peciolos de 4 a $8 \mathrm{~cm}$ de largo, y 4.5 a 8 foliolos por hoja (Cuadro 2). Se localizaron en suelos con pH ácido a neutro (5.5 a 7.1). 
Lagunes-Espinoza et al.: Diversidad morfológica y concentración de proteína de Lupinus, México

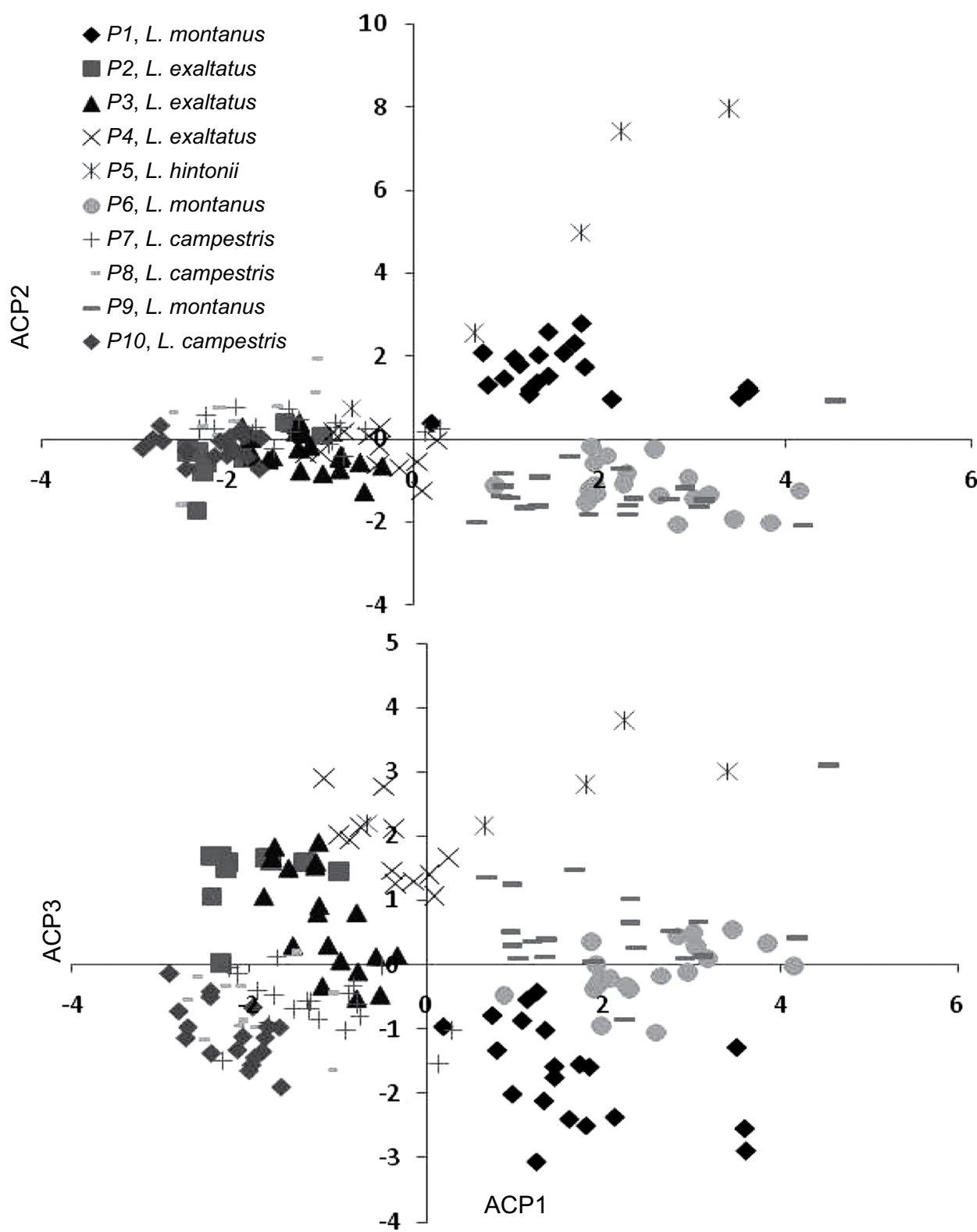

Fig. 2. Ubicación espacial de 10 poblaciones silvestres del género Lupinus colectadas en 2007, con base en los tres primeros componentes principales de variables morfológicas cuantitativas y cualitativas. ACP1: altura de planta, longitud de inflorescencia y número de foliolos por hoja. ACP2: largo y ancho de vaina y forma de estípulas. ACP3: largo de foliolos, forma de estípulas y tipo de tallo. 
Las características morfológicas de las poblaciones 2, 3, 4 correspondieron a Lupinus exaltatus; se ubicaron a partir de $2785 \mathrm{~m}$ s.n.m. en suelos con $\mathrm{pH}$ ácido y en bosques de P. montezumae con pastizal de Muhlenbergia sp. en el sotobosque. Presentaron una altura de planta de 0.3 a $1.0 \mathrm{~m}$, estípulas pequeñas, unidas ligeramente en la base de los peciolos, tallos huecos, inflorescencias de 1.5 a $13 \mathrm{~cm}$ de largo, 4 a 7 foliolos por hoja, largo y ancho promedio de foliolos de $2.7,3.8$ y $4.0 \mathrm{~cm}$ y $0.9,1.1$ y $1.1 \mathrm{~cm}$, respectivamente, vainas de 1.6 a $5 \mathrm{~cm}$ de largo y de 0.8 a $1.1 \mathrm{~cm}$ de ancho. En el año 2006, otro rodal de esta especie fue localizada a $2778 \mathrm{~m}$ s.n.m. en la misma área. A igual altitud se localizó también la existencia de $L$. campestris.

Finalmente, las poblaciones 6 y 9 se identificaron como L. montanus con altura de planta de 1.2 a $2.4 \mathrm{~m}$, diámetro del tallo principal de 9 a $35 \mathrm{~mm}$, largo de inflorescencias de 7 a $35 \mathrm{~cm}$, de 10.5 a 15 foliolos por hoja, largo y ancho de foliolos de 3.5 a $9.1 \mathrm{~cm}$ y de 0.5 a $1.3 \mathrm{~cm}$, respectivamente, estípulas grandes, envainantes y unidas en la base. Estas dos poblaciones (6 y 9) se encontraron en bosques de $A$. religiosa y $P$. hartwegii, en altitudes de 3080 a $3275 \mathrm{~m}$ (Cuadro 1). Otros rodales de $L$. montanus fueron observados durante el recorrido en el año 2006, a altitudes superiores a los $3400 \mathrm{~m}$, en suelos con $\mathrm{pH}$ ácido (4.9) y asociadas a bosque de $P$. montezumae con pastizal de Muhlenbergia sp. en el sotobosque; pero también se observaron en presencia de $P$. rudis y A. religiosa (Cuadro 1).

Concentración de proteína

Se cuantificaron altas proporciones de proteína cruda en las semillas de 16 localidades colectadas en los años 2006 y 2007 (Cuadro 3). La concentración promedio entre especies varió de 328 a $481 \mathrm{~g} \mathrm{~kg}^{-1} \mathrm{MS}$ (Cuadro 3). Entre poblaciones de una misma especie el intervalo fue más amplio. La mayor variación se observó entre las número 7, 8 y 10 de L. campestris (339 a $481 \mathrm{~g} \mathrm{~kg}^{-1} \mathrm{MS}$ ); para L. montanus, 2006 y 2007, fue de 339 a $420 \mathrm{~g} \mathrm{~kg}^{-1} \mathrm{MS}$; para las de L. exaltatus de 317 a $404 \mathrm{~g} \mathrm{~kg}^{-1} \mathrm{MS}$ y para L. hintonii de 299 a $356 \mathrm{~g} \mathrm{~kg}^{-1} \mathrm{MS}$; siendo la población 16 (L. exaltatus) y la 10 (L. campestris) las que presentaron, en este orden, la más baja $\left(317 \mathrm{~g} \mathrm{~kg}^{-1} \mathrm{MS}\right) \mathrm{y}$ la más alta (481 $\left.\mathrm{g} \mathrm{kg}^{-1} \mathrm{MS}\right)$ concentración de proteína en la semilla.

\section{DISCUSIÓN}

Las especies de Lupinus son un componente de la biodiversidad natural en la región de los Valles de Libres y Serdán del estado de Puebla, abundantes en el ecosistema forestal, tanto en el sotobosque como en claros del bosque, donde forman 
Cuadro 3. Medias e intervalos de variación de la concentración de proteína en la semilla de poblaciones de Lupinus spp. colectadas en la región de estudio.

\begin{tabular}{|c|c|c|c|c|c|}
\hline \multirow[t]{2}{*}{$\begin{array}{l}\text { Altitud } \\
\text { m s.n.m. }\end{array}$} & \multirow[t]{2}{*}{ Población } & \multirow[t]{2}{*}{ Especie } & \multirow[t]{2}{*}{$\begin{array}{l}\text { Año de } \\
\text { colecta }\end{array}$} & \multicolumn{2}{|c|}{$\begin{array}{l}\text { Concentración de proteína } \\
\qquad\left(\mathrm{g} \mathrm{kg}^{-1} \mathrm{MS}\right)\end{array}$} \\
\hline & & & & Media $^{\mathrm{a}}$ & Intervalo de variación ${ }^{a}$ \\
\hline 3115 & 1 & L. montanus & 2007 & 339 & $319.8-358.2$ \\
\hline 3080 & 6 & L. montanus & 2007 & 382 & $350.6-413.4$ \\
\hline 3275 & 9 & L. montanus & 2007 & 404 & $379.7-428.3$ \\
\hline 2910 & 11 & L. montanus & 2006 & 361 & $335.8-386.2$ \\
\hline 3292 & 12 & L. montanus & 2006 & 394 & $351.4-436.6$ \\
\hline 3423 & 13 & L. montanus & 2006 & 420 & $395.9-444.1$ \\
\hline 3471 & 14 & L. montanus & 2006 & 399 & $374.9-423.1$ \\
\hline \multirow[t]{2}{*}{3520} & 15 & L. montanus & 2006 & 400 & $375.6-424.4$ \\
\hline & & & Media $^{\mathrm{b}}$ & 387.4 & $361.0-420.0^{\mathrm{b}}$ \\
\hline 2865 & 2 & L. exaltatus & 2007 & 404 & $382.1-425.9$ \\
\hline 2842 & 3 & L. exaltatus & 2007 & 349 & $331.2-366.8$ \\
\hline 2785 & 4 & L. exaltatus & 2007 & 404 & $383.7-424.3$ \\
\hline \multirow[t]{2}{*}{2778} & 16 & L. exaltatus & 2006 & 317 & $306.0-328.0$ \\
\hline & & & Media $^{\mathrm{b}}$ & 368.5 & $317.0-404.0^{\mathrm{b}}$ \\
\hline 3080 & 5 & L. hintonii & 2007 & 328 & $299.5-356.5$ \\
\hline 3052 & 7 & L. campestris & 2007 & 339 & $312.1-365.9$ \\
\hline 2701 & 8 & L. campestris & 2007 & 349 & $326.2-371.8$ \\
\hline \multirow[t]{2}{*}{2615} & 10 & L. campestris & 2007 & 481 & $432.5-529.5$ \\
\hline & & & Media $^{b}$ & 372 & $339.0-481.0^{\mathrm{b}}$ \\
\hline
\end{tabular}

${ }^{a}$ Media e intervalo de variación al interior de una población. ${ }^{b}$ Media e intervalo de variación entre poblaciones de una misma especie.

grandes poblaciones, como es el caso de L. montanus, a partir de 2600 m s.n.m. Por el contrario, en las tierras agrícolas se observaron pocas plantas, tal vez a causa del laboreo continuo de los suelos de cultivo y de la presencia de roedores, factores que podrían tener un efecto negativo sobre la reserva de semillas (Maron y Simms, 1997). Además, sus inflorescencias son cortadas para las fiestas religiosas de la región, lo que conlleva a una presión adicional sobre los bancos de tales propágulos. 
Las poblaciones de Lupinus muestreadas en el presente estudio crecen en suelos con pH neutro a ácido, lo cual coincide con lo reportado en la Sierra Nevada (Alderete-Chávez et al., 2008) y en Europa y Sudamérica (Gladstones, 1970). Tal hecho les confiere una ventaja adaptativa sobre otras leguminosas ricas en proteína para colonizar áreas con este tipo de tierras de baja fertilidad.

Se observó una amplia variabilidad morfológica en las poblaciones de Lupinus estudiadas. La misma ya fue sugerida por Bermúdez-Torres et al. (2000) para México, en particular en el Eje Neovolcánico Transversal. Cox (1975) la atribuye a la gran plasticidad que presentan las especies de este género para adaptarse a los diferentes ambientes, y al alto grado de hibridación entre ellas. Esta diversidad puede ser aprovechada en la selección de atributos de interés agronómico.

De las especies de Lupinus identificadas en la región, la menos frecuente fue $L$. hintonii, encontrada en bosques de Abies religiosa, a una altitud de $3080 \mathrm{~m}$. Bermúdez-Torres et al. (2002) la reportan entre 2800 y $3200 \mathrm{~m}$ s.n.m. en bosques de pino-encino en el estado de Morelos, México, indicando que su morfología es muy cercana a la de L. montanus, pero tiene diferente composición de alcaloides. La presencia de esta última a partir de $3000 \mathrm{~m}$ s.n.m. en distintas regiones del Eje Neovolcánico Transversal es también reportada por Alderete-Chávez et al. (2008) y Bermúdez-Torres et al. (2009). L. montanus es la especie más frecuente en la región de estudio y crece en altitudes superiores a $2900 \mathrm{~m}$. Vargas (1984) la cita ya como planta dominante en el Parque Nacional Pico de Orizaba. También en la región forestal, pero a altitudes inferiores, se localizaron poblaciones de L. exaltatus, cuya presencia asimismo se registra en el Nevado de Colima, a partir de 1700 m (Ruiz-López et al., 2006). El otro taxon identificado en la región correspondió a L. campestris.

La concentración de proteína cuantificada en la población 10 de L. campestris (481 $\mathrm{g}$ de proteína $\mathrm{kg}^{-1} \mathrm{MS}$ ) es más alta que la reportada por Jiménez-Martínez et al. (2009) en semillas de la misma especie colectadas en el estado de Morelos (411.7 g $\mathrm{kg}^{-1} \mathrm{MS}$ ). En cambio, las encontradas en $L$. exaltatus fueron similares a las observadas por Ruiz-López et al. (2006) en poblaciones de esta planta colectadas en el Nevado de Colima, y a los valores reportados para especies domesticadas de lupinos: $L$. angustifolius, L. luteus, L. albus y L. mutabilis (300-440 $\mathrm{g} \mathrm{kg}^{-1} \mathrm{MS}$ ) por Petterson (1998) y Sujak et al. (2006). En comparación con otras leguminosas de grano, las plantas estudiadas mostraron concentraciones de proteína superiores a las conocidas para lenteja (Lens culinaris, $267 \mathrm{~g} \mathrm{~kg}^{-1} \mathrm{MS}$ ), haba (Vicia faba, $300 \mathrm{~g} \mathrm{~kg}^{-1} \mathrm{MS}$ ) y frijol (Phaseolus vulgaris, $290 \mathrm{~g} \mathrm{~kg}^{-1} \mathrm{MS}$ ) y semejantes a las de la soya (Glycine max, $405 \mathrm{~g} \mathrm{~kg}^{-1} \mathrm{MS}$ ) (Cubero y Moreno, 1983; Favier et al., 1995). También se observó variación en los contenidos de proteína entre las poblaciones de una misma especie. Jiménez et al. (1991) reportan 
diferencias entre sitios para contenidos de proteína en la semilla de L. albus, relacionadas con factores de fertilidad del suelo y ambientales como la temperatura. En otras especies de Lupinus nativas de Europa (Sujak et al., 2006), la concentración de proteína de la semilla varía de 295 a $482 \mathrm{~g} \mathrm{~kg}^{-1} \mathrm{MS}$; en $L$. albus de 351 a $376 \mathrm{~g} \mathrm{~kg}^{-1} \mathrm{MS}$, en $L$. angustifolius de 295 a 356 y en L. luteus de 447 a $482 \mathrm{~g} \mathrm{~kg}^{-1}$ MS., intervalos similares a los observados para las plantas mexicanas en nuestro estudio.

Recientemente diversos grupos de investigación han realizado estudios nutricionales sobre especies de Lupinus nativas de nuestro país (Przybylak et al., 2005; Rodriguez-Ambriz et al., 2005; Ruiz-López et al., 2006; Jiménez-Martínez et al., 2009), mostrando que presentan características nutricionales sobresalientes que pueden ser aprovechadas para la alimentación humana o animal. Estas plantas, colectadas en otras regiones del país y que han sido identificadas en la región de estudio con concentraciones similares o más elevadas de proteína, adaptación a diferentes altitudes y suelos ácidos de baja fertilidad, muestran una vía promisoria para su aprovechamiento sustentable, sea para restauración ecológica y/o como alternativa proteica o de componentes bioactivos para la alimentación humana o animal en nuestro país. Particularmente, estudios básicos sobre aspectos de agronomía que incluyan genética, fisiología, bioquímica, taxonomía entre otros, son elementos clave que permitirán la selección de la especie o las especies para el inicio del proceso de domesticación.

\section{AGRADECIMIENTOS}

Los autores agradecen el apoyo del M.C. Antonio López Castañeda en la elaboración de la figura 1 y al M.C. Arnulfo Triano Sánchez quién ayudó en la preparación de muestras para análisis proteico. Un reconocimiento especial al Dr. Alfonso Delgado Salinas, del Instituto de Biología de la Universidad Nacional Autónoma de México, por haber aceptado amablemente revisar la identificación taxonómica de los ejemplares botánicos. Igualmente, los autores agradecen a los revisores anónimos quienes con sus valiosas observaciones enriquecieron este documento.

\section{LITERATURA CITADA}

Alderete-Chávez, A., V. Espinosa, E. Ojeda, M. Ehsan, J. Pérez-Moreno, V. M. Cetina, D. A. Rodríguez-Trejo y N. de la Cruz-Landero. 2008. Natural distribution and principal characteristics of Lupinus in the oriental face of Tláloc Mountain in Sierra Nevada, México. J. Biol. Sci. 8: 604-609. 
Anónimo. 2000. SAS. Version 8.1. SAS Institute. Cary, USA.

Bermúdez-Torres, K., N. Robledo-Quintos, J. Martínez-Hernández, A. Tei y M. Wink. 2000. Biodiversity of the genus Lupinus in Mexico. In: van Santen, E., M. Wink, S. Weissman y P. Roemer (eds.). Lupin an ancient crop for the new millenium. Proceedings of the 9th International Lupin Conference. 20-24 June, 1999. Klink, Alemania. pp. 294-296.

Bermúdez-Torres, K., N. Robledo-Quintos, L. L. Barrera-Necha y M. Wink. 2002. Alkaloid profile of leaves and seeds of Lupinus hintonii C. P. Smith. Z. Naturforsch (C) 57: 243-247.

Bermúdez-Torres, K., J. Martínez, B. R. Figueroa, M. Wink y L. Legal. 2009. Activity of quinolizidine alkaloids from three Mexican Lupinus against the lepidopteran crop pest Spodoptera frugiperda. BioControl 54: 459-466.

Clements, J. C., B. J. Buirchel, H. Yang, P. M. C. Smith, M. W. Sweetingham y C. G. Smith. 2005. Lupin. In: Singh, R. J. y P. P. Jauhar (eds.). Genetic resources, chromosome engineering, and crop improvement grain legumes. Volume I. CRC Press, LLC. eBook. Boca Raton, Florida, USA.

Cox, B. J. 1975. Affinities of Lupinus prunophilus Jones and L. ammophilus Greene (Papilionaceae). Southwest. Nat. 20(2): 159-170.

Cruz, B. J., A. L. Martínez, R. Maldonado y G. Dávila. 1999. Cloning of a Lupinus campestris gamma conglutin fragment. In: van Santen, E., M. Wink, S. Weissman y P. Roemer (eds.). Lupin an ancient crop for the new millennium. Proceedings of the 9th International Lupin Conference. 20-24 June, 1999. Klink, Alemania. pp. 63.

Cubero, J. I. y M. T. Moreno. 1983. Leguminosas de grano. Mundi-Prensa. Madrid, España. $359 \mathrm{pp}$.

Dunn, D. B. 1979. Lupinus. In: Rzedowski, J. y G. C. de Rzedowski (eds.). Flora fanerogámica del Valle de México. Volumen 1. Compañía Editorial Continental, S.A. México, D.F., México. pp. 326-338.

Dunn, D. B. 1984. Genetic resources: Cytotaxonomy and distribution of New World lupin species. Proceedings of the 3th International Lupin Conference. 4-8 June, 1984. La Rochelle, Francia. pp. 68-85.

Dunn, D. B. y W. E. Harmon. 1977. The Lupinus montanus complex of Mexico and Central America. Ann. Missouri Bot. Gard. 64(2): 340-365.

Favier, J. C., J. L. Ripert, C. Toque y M. Feinberg. 1995. Répertoire général des aliments (table de compositions). 2a. ed. Institute National de la Recherche Agronomique Editions. Paris, Francia. 928 pp.

Gladstones, J. S. 1970. Lupins as crop plants. Field Crop Abst. 23: 123-148.

Gladstones, J. S. 1998. Distribution, origin, taxonomy, history and importance. In: Gladstones, J. S., J. S. Atkins y C. Hamblin (eds.). Lupinus as crop plants. Biology, production and utilization. CAB International. Oxon, UK. pp. 1-39.

Gutiérrez-Herrera, L., M. J. Cuervo y E. O. Ortiz. 2003. Regiones naturales y de planeación para el estado de Puebla. Análisis Económico 18(37): 257-296.

Huyghe, C. 1997. White lupin (Lupinus albus L.). Field Crops Res. 53: 147-160.

Jiménez, M. D., J. J. Cubero y A. Haro. 1991. Genetic and environmental variability in protein, oil and fatty acid composition in high-alkaloid white lupin (Lupinus albus). J. Sci. Food Agric. 55(1): 27-35. 
Jiménez-Martínez, C., R. Campos-Mendiola, M. E. Sánchez-Espíndola, A. JiménezAparicio, G. Gutiérrez-López y G. Dávila-Ortiz. 2009. Microstructural changes in Lupinus campestris seed in response to three thermal debittering treatments. J. Sci. Food Agric. 89(14): 2399-2404.

Jiménez-Martínez, C., H. Hernández-Sánchez y G. Dávila-Ortiz. 2003. Lupines: an alternative for debittering and utilization in foods. In: Gutiérrez-López, G. F. y G. V. Barbosa-Cánovas (eds.). Food Science and Food Biotechnology. Capítulo 11. CRC Press, LLC. eBook. Boca Raton, Florida, USA.

Jones Jr. J. B., B. Wolf y H. A. Mills. 1991. Plant analysis handbook: a practical sampling, preparation, analysis and interpretation guide. Micro-Macro Publishing. Atenas, Grecia. 357 pp.

Lewis, G. P., B. Schrire, B. Mackinder y M. Lock (eds.). 2005. Legumes of the world. Royal Botanic Gardens. Kew, UK. 577 pp.

Maron, J. L. y E. L. Simms. 1997. Effect of seed predation on seed bank size and seedling recruitment of bush lupine (Lupinus arboreus). Oecologia 111: 76-83

McVaugh, R. 1987. Lupinus. In: Anderson, W. (ed.). Flora Novo-Galiciana. Vol. 5. Leguminosae. The University of Michigan Press. Ann. Arbor., USA. pp. 580599.

Molumeli, A. P., V. Espinosa, M. Ehsan, S. B. Valdez, E. Ojeda, V. M. Cetina, A. AldereteChávez, N. de la Cruz-Landero y K. Santamaría. 2008. Lupines-invaded pine forests and cultivated scrublands in volcanic ash soils in Mexico: dry-sieved aggregation and instability indices. Int. J. Bot. 4(4): 390-405.

Petterson, D. S. 1998. Composition and food uses of Lupinus. In: Gladstones, J. S., C. A. Atkins y J. Hamblin (eds.). Lupinus as crop plant. Biology, production and utilization. CAB International. Oxon, UK. pp. 353-384.

Planchuelo, A. M. 1994. Wild lupin distribution and its implications as germplasm resources. In: Neves Martins, J. M. y M. L. Beirao da Costa (eds.). Advances in lupin research. Proceedings of 7 th International Lupin Conference. Evora, Portugal. Technical University of Lisbon. Lisboa, Portugal. pp. 65-69.

Przybylak, J. K., D. Ciessiolka, W. Wysocka, P. M. García-López, M. A. Ruiz-López, W. Wysocki y K. Gulewicz. 2005. Alkaloid profiles of Mexican wild lupin and an effect of alkaloid preparation from Lupinus exaltatus seeds on growth and yield of paprika (Capsicum annuum L.). Ind. Crop Prod. 21: 1-7.

Rodríguez-Ambriz, S. L., A. L. Martínez-Ayala, F. Millán y G. Dávila-Ortiz. 2005. Composition and functional properties of Lupinus campestris protein isolates. Plant Foods Hum. Nutr. 60: 99-107.

Ruiz-López, M. A., M. R. Rodríguez y S. Navarro P. 2006. Evaluación químico-nutricional de Lupinus exaltatus Zucc., del Nevado de Colima, México, como fuente potencial de forraje. Interciencia 31(10): 758-761.

Ruiz-Moreno, J. J., M. A. Ruiz-López y J. F. Zamora-Natera. 2000. The genus Lupinus: taxonomy and distribution in Jalisco, Mexico. In: van Santen, E., M. Wink, S. Weissman y P. Roemer (eds.). Lupin an ancient crop for the new millennium. Proceedings of the 9th International Lupin Conference. 20-24 June, 1999. Klink, Alemania. pp. 297-300. 
Sousa, S. M. y A. Delgado S. 1998. Leguminosas mexicanas: fitogeografía, endemismo y orígenes. In: Ramamoorthy, T. P., R. Bye, A. Lot \& J. Fa (comp.). Diversidad biológica de México: orígenes y distribución. Instituto de Biología, Universidad Nacional Autónoma de México. México, D.F., México. pp. 449-500.

Sujak, A., A. Kotlarz y W. Strobel. 2006. Compositional and nutritional evaluation of several lupin seeds. Food Chem. 98: 711-719.

Vance, C. P. 2001. Symbiotic nitrogen fixation and phosphorus acquisition. Plant nutrition in a world of declining renewable resources. Plant Physiol. 127: 390-397.

Vargas, M. F. 1984. Parques nacionales de México y reservas equivalentes; pasado, presente y futuro. Colección: Grandes problemas nacionales. Serie "Los Bosques de México". Instituto de Investigaciones Económicas, Universidad Nacional Autónoma de México. México, D.F., México. 266 pp.

Villaseñor, R. J. L. y F. J. Espinosa. 1998. Catálogo de malezas de México. Ediciones Científicas Universitarias. Serie Texto Científico Universitario. Universidad Nacional Autónoma de México, Consejo Nacional Consultivo Fitosanitario y Fondo de Cultura Económica. México, D.F., México. 449 pp. 